\title{
Electron overheating during field emission from carbon island films due to phonon bottleneck effect
}

\author{
A. V. Arkhipov, A. M. Zhurkin, O. E. Kvashenkina, V. S. Osipov, P. G. Gabdullin \\ Peter the Great St. Petersburg Polytechnic University, St. Petersburg, Russia \\ arkhipov@rphf.spbstu.ru
}

PACS 79.70.+q; 79.60.Jv; 81.05.uj; 81.07.b

DOI 10.17586/2220-8054-2018-9-1-110-113

\begin{abstract}
The paper discusses a possible model of low-field electron emission that could be applicable to carbon island films on silicon. Such films were recently showed to have emission thresholds as low as $0.4-1.5 \mathrm{~V} / \mu \mathrm{m}$. Discontinuity of the film - and not the presence of field-enhancing morphological features or low-workfunction spots - seems to be the necessary condition for good emission capability. We suggest a hot-electron emission model with emission center representing a single isolated nanosized island of $s p^{2}$ carbon having the properties of a quantum dot. Quantization of its electron energy spectrum determines electron/phonon decoupling ("phonon bottleneck" effect) and long electron relaxation times, which makes emission the dominating option for hot electrons of sufficient energy injected in the island. The consequences of this suggestion are quantitatively considered for typical experimental situation.
\end{abstract}

Keywords: low-field electron emission, carbon films, quantum dots, electron-phonon interaction, "phonon bottleneck" effect.

Received: 15 June 2017

Revised: 31 October 2017

\section{Introduction and problem formulation}

In our previous works [1-3], we observed field electron emission with threshold fields as low as $0.4-1.5 \mathrm{~V} / \mu \mathrm{m}$ from island films of graphitic carbon deposited on $\mathrm{Si}$ wafers. Comprehensive investigations performed with $\sim 50$ samples did not reveal the presence of either high-aspect morphological features or low-work-function spots, which excludes employment of many known emission facilitation mechanisms for explanation of the experimental results. Typically, the films with the best emission properties were composed of $s p^{2}-\mathrm{C}$ islands with lateral size $d \approx 10 \mathrm{~nm}$ and height $h \approx 3 \mathrm{~nm}$. The films were deposited on oxidized Si wafers, so that some of the islands had ohmic contact with the substrate while others were separated by Schottky barriers. Work function for the islands was measured by different methods which gave similar values $e \varphi \approx 4.7 \mathrm{eV}$. The difference in work function between the islands and the surrounding open substrate areas did not exceed $1 \mathrm{eV}$. We have to suggest a model of low-field electron emission relevant to this object.

\section{Emission model}

\subsection{General scheme}

We propose for consideration the following version of hot-electron emission model (Fig. 1). The emission center (EC) represents one of the carbon islands insulated from the substrate. The island is positively charged, so that its electric potential relative to the substrate $\left(+\phi_{E C}\right)$ approximately matches the work function value $e \varphi$. Hot electrons are injected in the EC from Fermi level of another island that has ohmic contact with the substrate. Electron transfer via a chain of intermediary islands is also possible. Energy of electrons injected in the EC is close to the local vacuum level, which secures high probability of their emission. External field applied to the cathode suffices to remove emitted electrons. This scheme has two obvious weak points.

1) Steady-state maintenance of the EC positive potential and charge needs to be explained. Some, even if a minor, part of injected hot electrons would fail to be emitted and accumulate. They should be removed from the EC's Fermi level against the action of the EC's own electric field. One possible solution of this problem we associate with thermoelectric effect, which may have huge magnitude at nanoscale, due to the peculiar character of phonon drag induced by ballistic phonon flow [4]. This issue is left beyond the limits of this paper and has been discussed elsewhere [5].

2) Energy relaxation times for hot electrons injected in the EC must be sufficient to allow their ballistic transport to vacuum boundary and emission. Further discussion addresses this problem. 


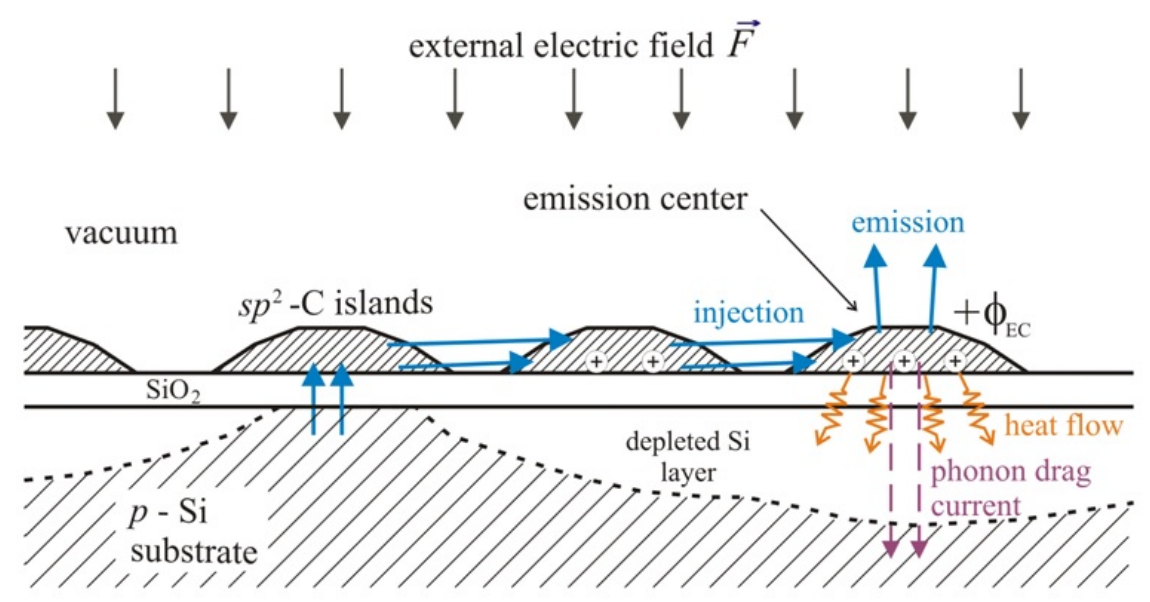

FIG. 1. To the mechanism of low-field emission from $s p^{2}$ carbon island films

\subsection{Hot-electron relaxation slowing}

Obstructed energy dissipation processes are a typical feature for many low-dimensional and nanosized objects with discrete energy spectra. Slowing of hot-electron cooling can be determined by the so-called "phonon bottleneck" effect [6,7]. If spacing between allowed energy levels exceeds the maximum phonon energy $(\sim 35 \mathrm{meV}$; given by Debye temperature $\left.\Theta_{D}\right)$, direct emission of phonons is prohibited, and hot electron relaxation goes via slower processes. Relaxation times as large as $\tau=0.1-1$ ns have been observed for semiconductor QDs [8,9]. This phenomenon attracts substantial interest because of its possible applications in solar batteries, infrared sensors, etc. Experimental information on electron relaxation rates in graphene and graphitic QDs is not so abundant (see, for instance in the review [10]). Though, the corresponding effects are expected to be "especially efficient owing to the unique properties of graphene: fast carrier-carrier scattering dominates over electron-phonon scattering" [10]. Among $s p^{2}$-carbon nanomaterials capable of low-field electron emission [11], evidence of suppressed electron-phonon interaction were reported for NPC $[12,13]$.

The regarded experimental situation satisfies both main requirements set by the basic theory for realization of the "phonon bottleneck". Maximum quantization gaps in energy spectra of QD of the given size $(10 \times 10 \times 3 \mathrm{~nm})$ were estimated as $\approx 200 \mathrm{meV} \gg k_{B} \Theta_{D}\left(k_{B}\right.$ is Boltzmann constant). The second condition requires hot electron density $\sim 10^{18} / \mathrm{cm}^{3}$ or higher [7]. In an island of the considered dimensions, this requirement would be fulfilled for current (in Amps) of injected hot electrons $I \geq 5 \cdot 10^{-20} / \tau$ - for instance, starting from $I=1 \mathrm{nA}$ for relaxation time as low as $\tau=50 \mathrm{ps}$.

The presented argumentation allows us to assume that hot electron relaxation time in the studied island films is much greater than that in bulk materials or continuous films. Consequences of this assumption for the emission process are discussed below. For quantitative estimates, relaxation time value $\tau=1 \mathrm{~ns}$ (determined in [8] for semiconductor QDs) will be used - because of the absence of experimental information more relevant for the considered object.

\subsection{Electron thermalization and emission}

Suppression of electron-phonon interaction could make either emission or scattering at other charge carriers the most probable option for hot electrons injected in the EC. In the latter case, hot-electron energy will be distributed among the EC free carriers to increase their temperature $T_{e}$ above the temperature of the lattice. We can roughly estimate it as:

$$
T_{e} \approx \frac{I \phi_{E C} \tau}{\frac{3}{2} k_{B} N},
$$

where $I$ is the current of injected hot electrons with excessive energy $e \phi_{E C}$; denominator is the heat capacity of EC electron subsystem expressed via the number of "intrinsic" charge carriers $N$ in the EC. Correct calculation of this number for a given EC requires knowledge of the density-of-states (DOS) function which is discrete and depends on the EC shape, dimensions, surface states' termination, etc [14]. These data are unavailable. For approximate estimates made for an "average" EC, we will use macroscopic formulae giving a smooth envelope for DOS functions relating to many different ECs [14]. For similar reason, we will use the simplest DOS formula for single-layer graphene, understanding that it is fully correct only near the Dirac point. 
In these assumptions, the number of mobile charge carriers may be calculated from the density of conduction electrons in graphene $n_{e}$ (per area unit):

$$
N=\frac{2 V \cdot n_{e}}{b}
$$

where $V \approx h d^{2}$ is the island's volume, $b \approx 0.335 \mathrm{~nm}$ is interplanar distance in graphite; factor 2 accounts for both holes and electrons.

Density $n_{e}$ can be obtained by integration of Fermi distribution:

$$
n_{e}=\int_{0}^{\infty} \frac{\nu(E) d E}{1+\exp \left(E / k_{B} T_{e}\right)},
$$

where $\nu(E)$ is energy density of allowed states; $E=0$ corresponding to Fermi level. Ignoring the fine details of the distribution, we can use the known formula for graphene:

$$
\nu(E)=\frac{g_{S} g_{V}}{2 \pi \hbar^{2} \nu_{F}^{2}}|E|
$$

Here, $v_{F} \cdot \approx 10^{6} \mathrm{~m} / \mathrm{s}$ is Fermi velocity, $g_{S}$ and $g_{V}$ are spin and valley degeneracy factors. The modulus signifies equivalent distributions for electrons and holes. Finally, we come to:

$$
T_{e}=\left(\frac{8 I \phi_{E C} \tau b \hbar^{2} \nu_{F}^{2}}{\pi g_{V} g_{S} V k_{B}^{3}}\right)^{1 / 3}
$$

For the previously assumed numeric values and typical emission (injection) current from on $\mathrm{EC} I=1 \mu \mathrm{A}$, the formula gives physically implausible electron temperature $T_{e} \approx 1.7 \cdot 10^{4} \mathrm{~K}$. At such temperatures, the current of thermionic emission would be $\gg I$. This disagreement could be formally resolved by introduction in (1) of a Nottingham heat term determined by the excess of emitted electrons' mean energy over the local Fermi level. Thus, the model (1) - (5) for injection current $I=1 \mu \mathrm{A}$ describes emission process as thermionic emission driven by greatly increased electron temperature in the EC. Notably, broad energy distributions of electrons field-emitted by carbon films have been described in literature (for instance, in [15]), they corresponded to effective emitter temperatures as high as $\sim 10^{3} \mathrm{~K}$.

Formula (5) describes very slow dependence of $T_{e}(I)$ which would be additionally slowed by the account of Nottingham cooling. This can witness of possible relevance of the depicted emission mechanism over a wide range of current values. For yet lower values of injection current electron energy distributions can be expected to deviate from quasi-equilibrium Fermi law. Density of states near Fermi level in graphitic carbon is low; hence electron temperature reduction will result in a rapid decrease of the carrier density. This will slow down thermalization of hot electrons via electron-electron scattering, the effect further enhanced by large difference in effective masses between hot and thermalized carriers. Thus, injected hot electrons emission can have greater probability than thermalization, and electron population would split in two separate groups. In this regime, emitted electrons could have relatively narrow energy distribution near the highest "phonon bottleneck" position.

\section{Acknowledgements}

The work was supported by State Assignment for Higher Education Institutions (basic part), project 3.5469.2017.

\section{References}

[1] Arkhipov A.V., Gabdullin P.G., Krel S.I., Mishin M.V., Shakhmin A.L., Gordeev S.K., Korchagina S.B. Field-induced electron emission from graphitic nano-island films at silicon substrates. Fuller. Nanotub. Car. N., 2012, 20(4-7), P. 468-472.

[2] Arkhipov A.V., Gabdullin P.G., Gordeev S.K., Zhurkin A.M., Kvashenkina O.E. Photostimulation of conductivity and electronic properties of field-emission nanocarbon coatings on silicon. Tech. Phys., 2017, 62(1), P. 127-136.

[3] Andronov A.N., Shkitun P.A., Budylina E.V., Gabdullin P.G., Gnuchev N.M., Kvashenkina O.E., Arkhipov A.V. Characterization of thin-film carbon field emitters. Tech. Digest 30-th Int. Vac. Nanoelectr. Conf. (IVNC'2017), Regensburg, Germany, 2017 , P. $276-277$.

[4] Eydelman E.D., Vul' A.Ya. The strong thermoelectric effect in nanocarbon generated by ballistic phonon drag of electrons. J. Phys. Condens. Matter, 2007, 19(7), P. 266210-266223.

[5] Arkhipov A.V., Gabdullin P.G., Kvashenkina O.E., Osipov V.S. Nanoscale phonon drag as a part of low-field electron emission mechanism for nanocarbons. Tech. Digest 30-th Int. Vac. Nanoelectr. Conf. (IVNC'2017), Regensburg, Germany, 2017, P. 28-29.

[6] Inoshita T., Sakaki H. Electron-phonon interaction and the so-called phonon bottleneck effect in semiconductor quantum dots. Physica B, 1996, 227(1-4), P. 373-377.

[7] Nozik A.J. Spectroscopy and hot electron relaxation dynamics in semiconductor quantum wells and quantum dots. Annu. Rev. Phys. Chem., 2001, 52, P. 193-231.

[8] Pandey A., Guyot-Sionnest P. Slow electron cooling in colloidal quantum dots. Science, 2008, 322, P. 929-932. 
[9] Tisdale W.A., Williams K.J., Timp B.A., Norris D.J., Aydil E.S., Zhu X.-Y. Hot-electron transfer from semiconductor nanocrystals. Science, 2010, 328, P. 1543-1547.

[10] Zhu S., Song Y., Zhao X., Shao J., Zhang J., Yang B. The photoluminescence mechanism in carbon dots (graphene quantum dots, carbon nanodots, and polymer dots): Current state and future perspective. Nano Res., 2015, 8(2), P. 355-381.

[11] Arkhipov A., Davydov S., Gabdullin P., Gnuchev N., Kravchik A., Krel S. Field-induced electron emission from nanoporous carbons. J. Nanomater, 2014, P. 190232.

[12] Popov V.V., Gordeev S.K., Grechinskaya A.V., Danishevskii A.M. Electrical and thermoelectric properties of nanoporous carbon. Phys. Solid State, 2002, 44(4), P. 789-792.

[13] Veinger A.I., Shanina B.D., Danishevskii A.M., Popov V.V., Gordeev S.K., Grechinskaya A.V. Electrophysical studies of nanoporous carbon materials prepared of silicon carbide powders. Phys. Solid State, 2003, 45(6), P. 1197-1206.

[14] Abdelsalam H., Espinosa-Ortega T., Luk'yanchuk I. Electronic and magnetic properties of graphite quantum dots. Low Temp. Phys., 2015, 41(5), P. 508-513.

[15] Reich K.V., Eidelman E.D., Vul' A.Ya. Determination of temperature difference in carbon nanostructures in field emission. Tech. Phys., 2007, 52(7), P. 943-946. 